\title{
Tumefactive multiple sclerosis in a young patient with hemiplegia and seizures associated with radiological appearance mimicking cerebral tumour
}

\author{
${ }^{1} \mathrm{~K}$ Khadjooi, ${ }^{2} \mathrm{G}$ Esposito, ${ }^{3} \mathrm{C}$ Dimopoulos \\ ${ }^{1}$ Department of Neurosciences, University Hospitals Coventry \& Warwickshire, Coventry, UK; ${ }^{2}$ Department of Medicine, Hull Royal \\ Infirmary, Kingston-upon-Hull, UK; ${ }^{3}$ Department of Medicine, Scarborough Hospital, Scarborough, UK
}

\begin{abstract}
Multiple sclerosis (MS) is the most common inflammatory demyelinating disease, affecting approximately one million adults worldwide. This paper describes a young female patient who presented to the acute medical team with generalised tonic-clonic seizures and right hemiplegia on a background of MS. Radiological imaging revealed a large ring enhancing lesion exerting mass effect. We describe an unusual form of MS and discuss diagnostic pitfalls of tumefactive demyelinating lesions which can be mistaken for brain tumours and abscesses.
\end{abstract}

KEYWORDS Tumefactive multiple sclerosis, tumour-like lesion, tumefactive demyelinating lesion

DECLARATION OF INTERESTS A poster of this case study was presented at the Society for Acute Medicine Conference in the UK in 2010.
Correspondence to K Khadjooi Department of Neurosciences, University Hospitals

Coventry \& Warwickshire, Clifford Bridge Road

Coventry CV2 2DX, UK

tel. +44 (0)24 76964000 e-mail kayvan@nhs.net

\section{CASE REPORT}

A 36-year-old woman was admitted as an emergency to a district general hospital with three short episodes of generalised tonic-clonic seizure. She gave a history of increasing right-sided weakness, slurred speech, facial asymmetry and unsteadiness over the past three weeks. Her past medical history consisted of multiple sclerosis (MS), diagnosed in 1998 with optic neuritis, but she was asymptomatic since 1999 and refused interferon therapy. On admission she was in the post-ictal phase of her third seizure with Glasgow Coma Scale of 9/15. She was slightly tachycardic and the rest of observations were within the normal range. Full neurological examination revealed expressive dysphasia, right upper motor neuron facial weakness, dense right hemiplegia (Medical Research Council scale $=0 / 5$ ), and a right upgoing plantar reflex. Except for a white blood cell count of $14.3 \times 109 / \mathrm{L}$, all other basic investigations were normal.

An urgent computerised tomography (CT) scan of the brain showed a $3.8 \mathrm{~cm}$ cavitating mass within the left parietal lobe with extensive surrounding vasogenic oedema and moderate midline shift, presumed to be a brain tumour (Figure I). After consultation with the neurosurgical team, she was started on dexamethasone and further imaging was arranged. Magnetic resonance imaging (MRI) of the brain with gadolinium demonstrated multiple ring enhancing lesions in both cerebral hemispheres, with the largest in the left parietal region surrounded by fairly extensive oedema with some mass effect on the left lateral ventricle (Figures 2A and 2B).

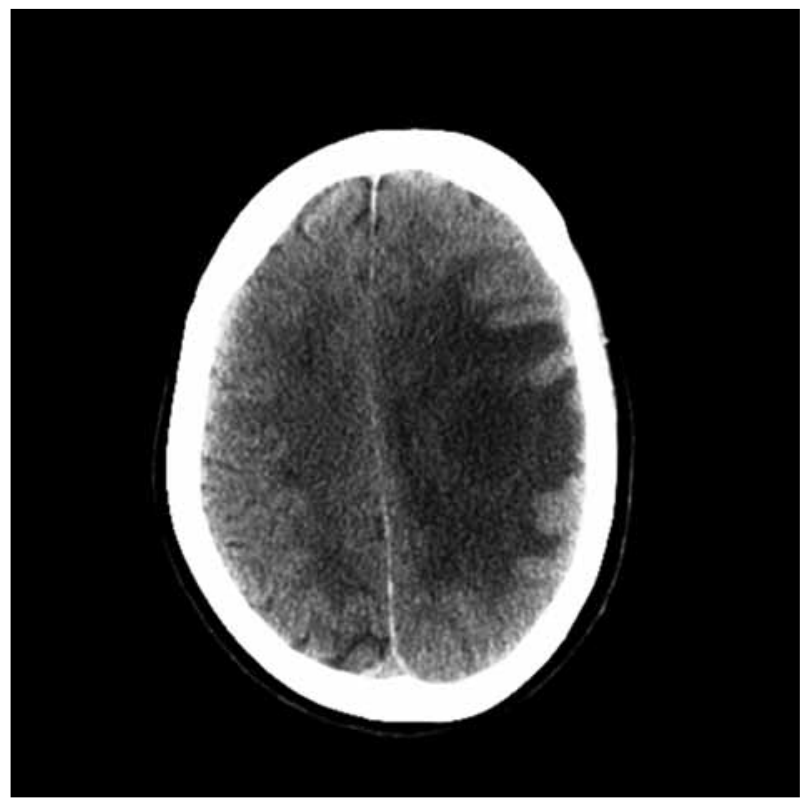

FIGURE I Computerised tomography (CT) scan of the brain of a 36-year-old woman admitted as an emergency following three short episodes of generalised tonic-clonic seizures with a history of increasing right sided weakness, slurred speech, facial asymmetry and unsteadiness over the past three weeks. The scan shows a moderate midline shift and within the left parietal lobe, extensive vasogenic oedema and a $3.8 \mathrm{~cm}$ cavitating mass presumed to be a brain tumour.

At the neurosurgical multi-disciplinary meeting, the differential diagnosis of tumefactive multiple sclerosis (TMS) or brain metastasis was made and it was advised to start the patient on methylprednisolone, monitor the response and look for a possible primary malignancy, 

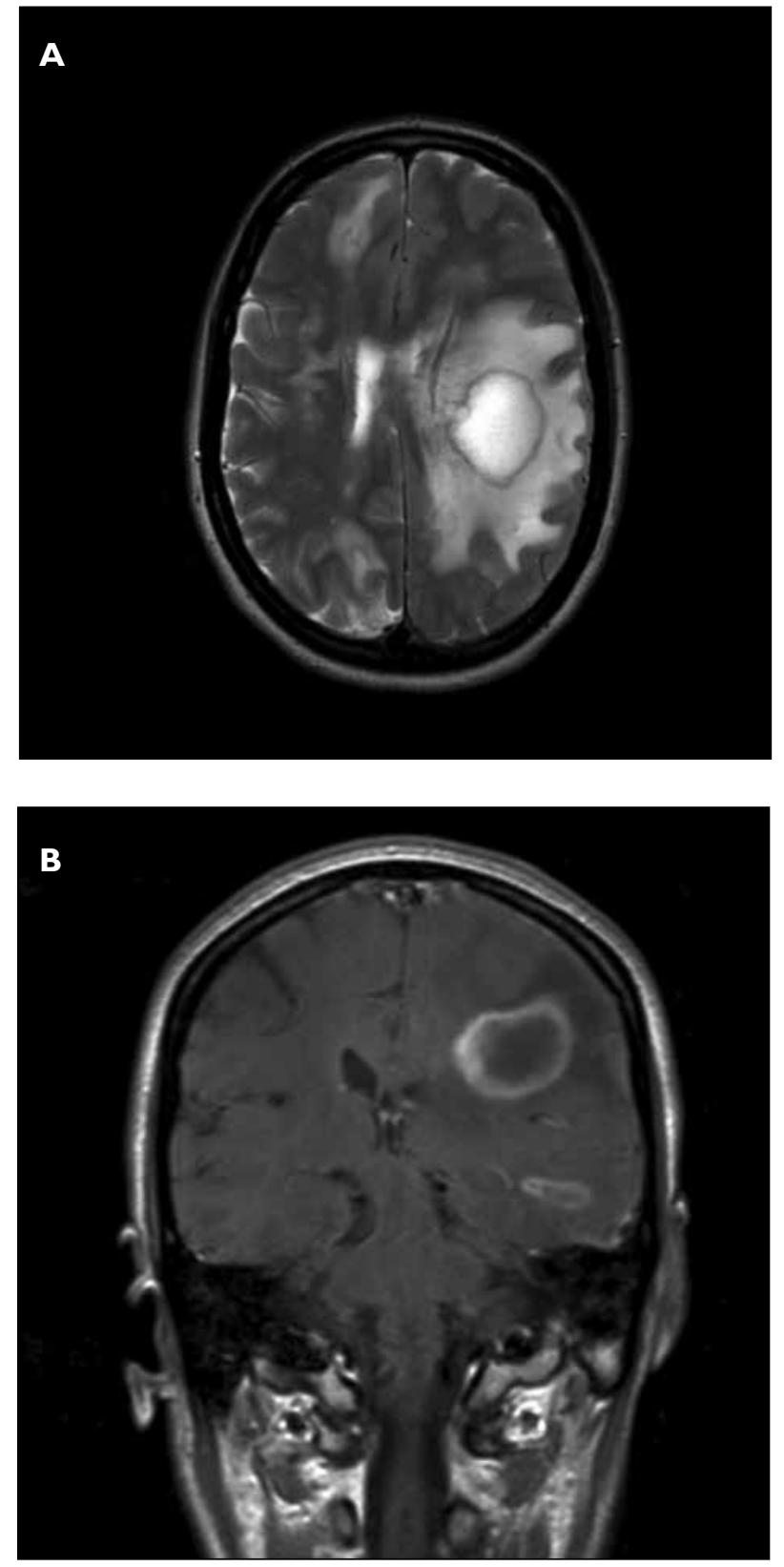

FIGURE 2 Gadolinium enhanced magnetic resonance image (MRI) scan of the brain. (A) Sagittal view: multiple ring enhanced lesions in both cerebral hemispheres. (B) Coronal view: the largest lesion in the left parietal region is surrounded by fairly extensive oedema and impinges on the left lateral ventricle.

although a brain biopsy might be needed. A mammogram and CT scan of the chest, abdomen and pelvis did not show any abnormalities. The patient made considerable improvement after two days of methylprednisolone and was transferred to the neurology ward in a tertiary centre. She continued to improve over the following days and at the end of week two was able to walk with a stick and the power on the affected limbs was $3 / 5$. Considering her previous history of MS, excellent response to steroids and the absence of clinical, biochemical, and radiological evidence of a primary malignancy, the diagnosis of TMS was felt to be the most likely diagnosis. Community physiotherapy was arranged on her discharge home.

\section{DISCUSSION}

Demyelinating plaques on MRI generally appear as multiple, well demarcated, homogenous, small ovoid lesions which lack mass effect and are often perpendicular to the long axis of the lateral ventricles.' The presence of tumefactive demyelinating lesions (TDL) on MRI can cause diagnostic difficulties. Differential diagnosis lies between demyelinating lesion and neoplasm, and accurate diagnosis influences further management. The precise assessment of such lesions at the first clinical manifestation of the disease is particularly important.

Tumefactive MS refers to an enhancing highly active MS lesion that may exert mass effect and be mistaken for a tumour. This is a large tumour-like lesion typically found in younger individuals with rapidly progressive neurological deficits. ${ }^{2}$ The incidence of TMS is estimated to be $I-2 / I, 000$ cases of MS and almost always occurs in patients with a prior diagnosis of MS. ${ }^{2}$ Symptoms are generally atypical for $M S$ and usually relate to the presence of a focal mass lesion. ${ }^{3}$ The largest case series $(n=168)$ described only $6 \%$ of cases as presenting with generalised tonic-clonic seizures, with the majority of cases presenting with focal neurological deficit.'

Although distinguishing TMS from central nervous system (CNS) malignancy is not possible purely on the basis of radiographic features, there are common MRI features of TDLs that will assist in differentiating them from other pathologies. These include variable degrees of mass effect and surrounding oedema, a T2 hypointense rim, venular enhancement, peripheral restriction on diffusion-weighted imaging and incomplete ring enhancement. ${ }^{4-7}$ Importantly, Kiriyama et al described venous dilatations on and around the TDL at MR angiography that can help facilitate diagnosis. ${ }^{4} \mathrm{Hydrogen}-\mathrm{I}$ MR spectroscopy can also be helpful in differentiating these lesions from neoplasms. ${ }^{2}$ Physiologic (permeability) and hemodynamic (blood volume) parameters on perfusion CT have been used to differentiate TDLs from high grade gliomas. Due to lack of neoangiogenesis and vascular endothelial proliferation, TDLs show lower permeability surface area product and lower blood volume as compared to high grade gliomas. ${ }^{8}$

An important role remains for diagnostic brain biopsy in some cases. Although greater appreciation of the clinical spectrum of this challenging disorder and use of other diagnostic techniques (i.e. visual evoked potentials and cerebrospinal fluid [CSF] oligoclonal bands) may obviate the need for biopsy in certain cases, brain biopsy is occasionally necessary to confirm the diagnosis, even in the presence of established MS.' In the largest published case series, the most common reasons why patients may 
have biopsy included atypical presentations (such as seizures or aphasia), older age, larger lesion size $>2.0 \mathrm{~cm}$, unusual appearances on MRI and associated mass effect.'

Initial smaller case studies suggested that prognosis for patients presenting with TDL was favourable and that in the majority of patients this was an isolated presentation. Lucchinetti suggests that in his case series (which only included biopsy proven cases), the majority (70\%) went on to develop definite multiple sclerosis.' It is interesting to note that in this cohort, a third already had MS and thus the true proportion of patients progressing to definite MS with a true first presentation with TDL is not clear.' Interestingly, however, the time between initial presentation with TDL and the second disease-defining event was 4.8 years, which is significantly longer than with other initial presentations of MS. The cause of this

\section{REFERENCES}

I Lucchinetti CF, Gavrilova RH, Metz I et al. Clinical and radiographic features of pathologically confirmed tumefactive multiple sclerosis. Brain 2008; I31:1759-75. doi:10.1093/brain/awn098

2 Ernst T, Chang L, Walot I et al. Physiologic MRI of a tumefactive multiple sclerosis lesion. Neurology 1998; 51:1486-88.

3 Paley RJ, Persing JA, Doctor A et al. Multiple sclerosis and brain tumor: a diagnostic challenge. J Emerg Med 1989; 7:24I-44. doi:I0.1016/0736-4679(89)90353-3

4 Kiriyama T, Kataoka H, Taoka T et al. Characteristic neuroimaging in patients with tumefactive demyelinating lesions exceeding $30 \mathrm{~mm}$.J Neuroimaging 20 I I; 2 I:e69-77. doi:I0.I I I /j.1552-6569.2010.00502.x

5 Malhotra HS, Jain KK, Agarwal A et al. Characterization of tumefactive demyelinating lesions using $M R$ imaging and in-vivo proton MR spectroscopy. Mult Scler 2009; 5:193-203. time difference is not certain.' Prognosis in terms of disease severity does not seem significantly different between those presenting with a TDL and those with typical MS presentations.'

\section{CONCLUSION}

Tumefactive MS is a rare but important form of a common neurological condition which can present as pseudoneoplasms of the nervous system ${ }^{10}$ and cause diagnostic difficulties due to its radiological similarity to other intra-cerebral pathologies, including brain tumours and abscesses. Awareness of this condition among nonneurologists is particularly important as it facilitates timely diagnosis, referral and treatment and avoids unnecessary investigations.
6 Selkirk SM, Shi J. Relapsing-remitting tumefactive multiple sclerosis. Mult Scler 2005; I I:73 I-34. doi: I0. I I9|/ 352458505ms I 2 /4cr

7 Jamroz-Wiśniewska A, Janczarek M, Belniak E et al. Tumour-like lesions in multiple sclerosis. Neurol Neurochir Pol 2008; 42: 16I-67.

8 Jain R, Ellika S, Lehman NL et al. Can permeability measurements add to blood volume measurements in differentiating tumefactive demyelinating lesions from high grade gliomas using perfusion CT? J Neurooncol 2010; 97:383-88. doi:| 0.1007/s I 1060-009-0030-2

9 Wattamwar PR, Baheti NN, Kesavadas C et al. Evolution and long term outcome in patients presenting with large demyelinating lesions as their first clinical event. J Neurol Sci 2010; 297:29-35. doi:10.1016/j.jns.2010.06.030

10 Donev K, Scheithauer BW. Pseudoneoplasms of the nervous system. Arch Pathol Lab Med 2010; 134:404-16. 